\title{
Cognitive Impairment in Schizophrenia: An Overview of Assessment and Management
}

\author{
Susmita Halder ${ }^{1}$, Akash Kumar Mahato ${ }^{2}$
}

\section{ABSTRACT}

Schizophrenia is a psychiatric illness characterized by gross disturbances in thought, perception and speech. The illness is heterogenous in terms of its manifestation as well as etiological factors. Over last two decades, evidence of cognitive impairment in schizophrenia and its impact on functioning and outcome in schizophrenia has been one of the predominant areas of research in schizophrenia. The present article gives an overview of cognitive impairment in schizophrenia, its nature and course as well as remediation strategies.

Keywords: Schizophrenia, cognitive impairment, remediation

Schizophrenia is a major psychiatric disorder characterized by a disruption in affective, cognitive and social domains, which results in compromised ability to adapt to a changing environment and to function adequately in the community. Over the past 20 years, there has been an increased recognition of the importance of cognitive impairments in schizophrenia (Sharma and Antonova, 2003). Even though modern antipsychotics and psychosocial treatment programs are effective in moderating symptoms and reducing the rate of relapses, most patients with schizophrenia do not function well outside structured, routine settings. Outcomes other than symptoms improvement and relapse prevention have not been well documented (Lehman, 1995; Hogarty \& Flesher, 1999). A majority of patients with schizophrenia interact in ordinary social settings in a conspicuous way and also do not manage an ordinary work very well. On average, only 10-30\% of patients with schizophrenia are employed at any time, and few these are able to maintain their vocation (Attkisson et al.1992). Many clinicians now claim that the reason is the serious cognitive dysfunction is the enduring core feature of schizophrenia.

Cognitive Impairment in Schizophrenia: Schizophrenia is often accompanied by gross and progressive impairment in different functional areas of a person. They are mostly seen in the form of - cognitive impairment (executive functions, information processing \& attention, --

1\&2 Assistant Professor in Clinical Psychology, Amity University, Rajasthan.

(C) 2015 I S Halder, A Mahato; licensee IJIP. This is an Open Access Research distributed under the terms of the Creative Commons Attribution License (http://creativecommons.org/licenses/by/2.0), which permits unrestricted use, distribution, and reproduction in any Medium, provided the original work is properly cited. 


\section{Cognitive Impairment in Schizophrenia: An overview of assessment and management}

--learning and memory), psychomotor activity and speech, thought process, perception, and abstraction. Among these, the neurocognitive impairment is gradually now being seen as an integral part of schizophrenia to the extent that, many researchers imply cognitive impairment as the core the disorder (Elvevag B, Goldberg TE., 2000). Extensive research in the field has suggested that the cognitive deficits associated with schizophrenia are not merely a consequence of psychotic symptoms or its treatment, but rather a distinct dimension of the illness. Perhaps one of the most challenging aspects of treating patients with schizophrenia is coping with the severe cognitive deficits that accompany the disease. These deficits are evident in nearly all individuals diagnosed with schizophrenia, and their impact on the employment, social relationships and living status of patients is devastating. The cognitive deficits not only impair motivation and insight about one's illness and treatment, they also negatively affect the therapeutic alliance.

Early Studies: In the 1960s, investigations into disrupted attentional functions started with systematic experimental investigations of reaction time parameters in the differentiation of schizophrenics from non- psychiatric individuals. Clinical research in the same period had shown that schizophrenic patients performed abnormally on measures of general intellectual functioning (Heaton \& Crowley, 1981). Indeed, it turned out that when a group of patients with schizophrenia are compared to healthy controls on a neuropsychological test battery, performance of the patients group was worse on almost all of the tests and comparable to the performance of patients with known neurological damage. However, a description of the symptoms, cognition and behavior of schiziphrenics in terms of the dichotomy 'organic' versus 'functional' has not proved very fruitful. Nowadays, schizophrenia is considered as a psychiatric disorder wth neuropsychological correlates. Those more acuratly characterizes the illness and enebles the investigation of specific brain- behavior relationships which underlie the variablity of symptoms and neurobehavioral systems which may be affected. The prevalence of neuropsychological deficits in schizophrenia has been widely documented; $50 \%-80 \%$ of patients with schizophrenia may be classified as brain damaged using actuarial methods developed in neurological populations (Kolb and Whishaw 1983).

However, there are some controversies over identifying patients, as having a cognitive deficit. That is whether all schizophrenics develop cognitive impairment? Some conceptualizations of this issue have relied on approaches from clinical neuropsychology that define cognitive impairment. These definitions are based on a performance deficit compared with a healthy control population, such as one standard deviation below the mean on one or more areas of cognitive function (Palmer et al 1997). The estimated percentage of schizophrenia patients who do not have a cognitive deficit by these definitions has varied from 27\% (Palmer et al 1997) to 55\% (Bryson et al 1993). 
The nature of cognitive impairment: Over the past 20 years a wealth of data has accumulated regarding the presence and stability of neuropsychological impairment in schizophrenia. The few published reviews range from quite positive (Kurtz et al. 2001), to somewhat positive (Twamley et al. 2003), to negative (Pilling et al. 2002). Schizophrenia patients exhibit widespread deficits in many domains, ranging from abnormalities in pre-attentional sensory processing to gross impairments in everyday functioning. The range of deficits in schizophrenia is extremely broad. Most recent neuropsychological research suggests that deficits are evident in following broad range of domains.

- Motor Skill

- Sensory Abilities

- Attention

- Learning

- Verbal ability,

- Complex perceptual skill,

- Abstraction and cognitive flexibility,

Though, several extensive reviews have described numerous neuropsychological deficits in patients with schizophrenia, the most consistently replicated deficits in schizophrenia patients have been observed on tasks measuring Attention/ Information- Processing, Learning, Memory, and Executive Functions. Impairments of schizophrenia patients on motor tasks are often attributable to medication effects on performance, which include both motor and cognitive side effects (Nuechterlein, 1986). Findings of impairment in basic language and visuo-spatial functions have been inconsistent, and deficits are typically reported on complex tasks that make significant demands on attention, or executive functions.

\section{ATTENTION, MEMORY AND EXECUTIVE FUNCTIONS:}

The most common cognitive impairments found in patients with schizophrenia are those of attention, memory and executive functions. Schizophrenia is characterized by deficits in attention and it has been claimed that these attention deficits are related to dysfunctional brain systems that underlie the pathophysiology of the disease (Robbins, 1990; Norman et al 1997). The characteristic symptoms of the attention deficits include perseveration (Cromwell and Dokecki, 1968; Goldman et al, 1991, 1992), over switching (Salzinger, 1973, 1983; Frith and Done, 1983, 1989), and inability to ignore irrelevant stimuli (Solomon et al, 1981; Anscombe, 1987). Attentional skills are disrupted by a wide variety of causes and attention is a critical underlying component of many cognitive functions.

Memory impairment is one of the most common cognitive problems in schizophrenia (Gourovitch and Goldberg, 1996). This type of impairment is usually long- term, debilitating, and difficult to treat (Chen and McKenna, 1996). Schizophrenia patients have been found to be impaired on their ability to recall, recognize, and learn both visual and verbal materials (Tamlyn 
et al, 1992; Paulsen et al 1995). These findings have been postulated to result from neuropathological abnormalities in the temporal- hippocampal area (Goldman et al, 1996). Increasing amount of research has investigated memory deficits in schizophrenia and studies have documented greater impairments in verbal memory than in other cognitive functions and these deficits do not appear to be accounted for by abstraction or attention (Gold et al., 1992; Saykin et al.1994).

Executive functions include the capacity to formulate goals, plan and organize goal- directed behavior, carry out goal directed behavior fully and effectively, and monitor and self- correct one's behavior needed. Schizophrenia patients have varying grades of impairment of executive processes, which result in difficulties during extended and multifaceted interpersonal interactions. Carter and Barch (2000) relate the neural basis of executive functions to the dorsolateral prefrontal cortex and anterior cingulated cortex, and show that functional imaging studies identified disturbances in other areas as well, particularly the temporolimbic region.

\section{THE COURSE OF COGNITIVE DEFICITS IN SCHIZOPHRENIA:}

There are several contrasting views on the course of cognitive function in schizophrenia. One course suggests that the disease process manifests itself as cognitive impairment that may be relatively profound and widespread at an early stage of development and is present subsequent to the onset of psychotic symptoms. These premorbidly compromised patients may have experienced early developmental stressors and/or a genetic predisposition leading to the observed cognitive deficits.

After an insidious onset, patients' intellectual functions become weaker and social skills become coarser.

A second view suggests that the cognitive deficits may become manifest concurrently with the onset of psychotic symptoms, resulting in a more circumscribed pattern of deficits that encompass the cognitive domains of executive function, attention and long term memory. This process is, however self limiting, as patients do not go on to a full blown dementia and this occurs in the presence of intellectual decline.

A third view thus suggests that while cognitive impairment may be concurrent with onset of symptoms, the debilitating cognitive deficits associated with the disease process may be relatively subtle, being restricted to the domains of executive function/ working memory and attention. It is unclear whether the deficits in this group precede symptoms or emerge concurrently (Weickert T W; Goldberg T E 2000)

However, it is clear that once the clinical manifestations of the illness become overt, a sharp decline in cognitive ability takes place in many patients (Goldberg T E. 2002). 


\section{HOW CAN WE TREAT COGNITIVE DEFICITS?}

Since schizophrenia may not be a single condition and its causes are not yet known, current treatment methods are based on both clinical research and experience.

Pharmacological treatments: Antipsychotic medications have been available since the mid1950s. They have greatly improved the outlook for individual patients. These medications reduce the psychotic symptoms of schizophrenia and usually allow the patient to function more effectively and appropriately. Antipsychotic drugs are the best treatment now available, but they do not "cure" schizophrenia or ensure that there will be no further psychotic episodes. The choice and dosage of medication can be made only by a qualified physician who is well trained in the medical treatment of mental disorders. The dosage of medication is individualized for each patient, since people may vary a great deal in the amount of drug needed to reduce symptoms without producing troublesome side effects. At present there are no drugs specifically approved for ameliorating impaired cognition in schizophrenia. Typical antipsychotic drugs do not improve cognition significantly (Harvey and Keefe, 2001). A number of new antipsychotic drugs (the so-called "atypical antipsychotics") have been introduced since 1990. Recent meta-analyses of atypical antipsychotic drugs suggest a significant, yet mild, cognitive improvement with atypical versus conventional antipsychotics (Harvey and Keefe, 2001; Woodward et al., 2002). Recent attention to the need for improving cognition in schizophrenia has included a conference sponsored by the U.S. Food and Drug Administration on developing standards for the approval of cognition-enhancing drugs (Buchanan, 2004). In addition, several large-scale, industrysponsored trials are underway, although the studies completed to date offer no specific guidance regarding cognitive-enhancing treatments in schizophrenia.

\section{Nonpharmacological treatments:}

Antipsychotic drugs have proven to be crucial in relieving the psychotic symptoms of schizophrenia - hallucinations, delusions, and incoherence - but are not consistent in relieving the behavioral symptoms of the disorder. Even when patients with schizophrenia are relatively free of psychotic symptoms, many still have extraordinary difficulty with communication, motivation, self-care, and establishing and maintaining relationships with others. It is with these psychological, social, and occupational problems that psychosocial treatments may help most. While psychosocial approaches have limited value for acutely psychotic patients, they may be useful for patients with less severe symptoms or for patients whose psychotic symptoms are under control. Numerous forms of psychosocial therapy are available for people with schizophrenia, and most focus on improving the patient's social functioning - whether in the hospital or community, at home, or on the job. Some of these approaches are described here.

Family Education: Very often, patients with schizophrenia are discharged from the hospital into the care of their family; so it is important that family members learn all they can about schizophrenia and understand the difficulties and problems associated with the illness. It is also 
helpful for family members to learn ways to minimize the patient's chance of relapse - for example, by using different treatment adherence strategies - and to be aware of the various kinds of outpatient and family services available in the period after hospitalization. Family "psychoeducation," which includes teaching various coping strategies and problem-solving skills, may help families deal more effectively with their ill relative and may contribute to an improved outcome for the patient.

Individual Psychotherapy: In individual psychotherapy, sessions may focus on current or past problems, experiences, thoughts, feelings, or relationships. By sharing experiences with a trained empathic person - talking about their world with someone outside it - individuals with schizophrenia may gradually come to understand more about themselves and their problems. They can also learn to sort out the real from the unreal and distorted. Recent studies indicate that supportive, reality-oriented, and cognitive-behavioral approaches that teach coping and problemsolving skills can be beneficial for outpatients with schizophrenia. However, psychotherapy is not a substitute for antipsychotic medication, and it is helpful once drug treatment first has relieved a patient's psychotic symptoms.

Self-Help Groups: Self-help groups for people and families dealing with schizophrenia are becoming increasingly common. Although not led by a professional therapist, these groups may be therapeutic because members provide continuing mutual support as well as comfort in knowing that they are not alone in the problems they face. Self-help groups may also serve other important functions. Families working together can more effectively serve as advocates for needed research and hospital and community treatment programs. Patients acting as a group rather than individually may be better to improve their social skills and interaction.

Rehabilitation : Broadly defined, rehabilitation includes a wide array of non-medical interventions for those with schizophrenia. Rehabilitation programs emphasize social and vocational training to help patients and former patients overcome difficulties in these areas. Programs may include vocational counselling, job training, problem-solving and money management skills, use of public transportation, and social skills training. These approaches are important for the success of the community-centered treatment of schizophrenia, because they provide discharged patients with the skills necessary to lead productive lives outside the sheltered confines of a mental hospital.

It may be mentioned that family education, individual psychotherapy or self help groups may not be a direct intervention on specific cognitive deficits, but are effective in improving outcome in terms of initiation and maintaining daily activities.

\section{COGNITIVE REHABILITATION:}

Cognitive deficiencies are a core feature of schizophrenia, and they have a dramatic effect on real-world functioning. Knowledge of an individual patient's cognitive strengths and weaknesses 
can help psychiatrists use techniques that are best suited to that particular patients. There are a variety of ways that psychiatrists can assess the cognition of their patients.. Although little is known at present about how best to treat cognitive deficits pharmacologically, a great deal of research is currently underway. Cognitive remediation therapies are also available to improve some cognitive skills. A focus on treating the cognitive deficits of patients with schizophrenia may be one of the best things that we can do to provide them with better real-world functioning and, thus, with better lives.

Cognitive rehabilitation is a form of intervention in which a series of procedures are applied by a trained practitioner to retain or alleviate problems due to deficits in underlying cognitive functions (Sohlberg and Mateer, 1989). It is a teaching process that targets areas of neuropsychological functioning involved in learning and basic day-to-day functioning. In response to the increased focus on cognitive deficits in schizophrenia, researchers and clinicians have developed efforts to systematically reduce these cognitive impairments. However, targeting of cognitive and neuropsychological deficits in schizophrenia for therapeutic interventions has so far been greatly neglected. Attempts at cognitive remediation have been relatively sparse. Neuropsychologists have contributed strongly to the development of the few remediation programs for schizophrenia patients.

There are two distinctly different approaches in cognitive therapy with schizophrenia patients. One focuses on cognitive content, while the other is process- oriented, emphasizing the correction of basic cognitive deficits. The field of cognitive rehabilitation of schizophrenia now includes individualized and group treatments, as well as computerized and non- computerized methods.

Basic methods of cognitive rehabilitation: The first step in developing a cognitive rehabilitation plan generally involves a comprehensive neuropsychological evaluation to determine the areas of cognitive deficits, as well as areas of cognitive strengths that can be used, especially for compensation techniques. This evaluation would also include an evaluation of mood and personality factors known to interact with cognitive functioning. Finally, the person's level of insight or awareness will be determined, since this is a critical factor in the success of rehabilitation.

\section{INTEGRATED PSYCHOLOGICAL THERAPY:}

This is a group-based cognitive remediation technique developed by Brenner et al (1994). This therapy focuses on a hierarchy of different skills, beginning with executive functioning through conceptual differentiation then social perception, verbal communication, social skills and problem solving.

Conclusion: As a core symptom, neuropsychological dysfunction specific to the disorder must be precisely defined, assessed and targeted for remediation if these patients are to be treated effectively. Because of the heterogeneity of the disorder, different treatments may be effective in 
certain subgroups, and certain cognitive abilities may be more responsive to intervention and more relevant to clinical outcome than others. There is much to accomplish in the treatment of this disabling disorder. Even though modern antipsychotics and psychosocial treatment programs are effective in moderating symptoms and reducing the role of relapses, most patients with schizophrenia do not function well outside structured settings. Effective techniques for normalizing and neutralizing cognitive impairments would be decisive additions to the treatment for patients with schizophrenia.

\section{REFERENCES:}

Attikisson, C ; Cook, J.; Karno, M.; Lehman, A.; McGlashan, T.H.; Meltzer, H.Y.; O'Connor, M.; Richardson, D.; Rosenblatt, A.; Wells, K.; Williams, J.; and Hohmann, A.A. 1992. Clinical research services. Schizophrenia Bulletin 18, (4), 561-626.

Brenner HD, Roder V, Hodel B, \& Corrigan P (1994). Integrated Psychological Therapy for Schizophrenic Patients. Toronto: Hogrefe and Huber.

Censists, D.M. et al. 1997. Neuropsychological evidence supporting a neurodevelopment model of schizophrenia: a longitudinal study. Schizophrenia Research, 24, 289- 98.

Elvevag B; Goldberg T. E. (2000). Cognitive impairment in schizophrenia is the core of the disorder. Critical Review of Neurobiology; 14(1):1-21.

Faith D; John J. B; Norman R; Frederick P. 1996. Neurocognitive deficits and social functioning in outpatients with schizophrenia. Schizophrenia Research; 21(2- 23) August, 75-83.

Goldberg TE, Bigelow LB, Weinberger DR, Daniel DG, Kleinman JE 1991. Cognitive and behavioral effects of the coadministration of dextroamphetamine and haloperidol in schizophrenia. Am J Psychiatry 148: $78 \square 84$.

Goldberg TE, Ragland JD, Torrey EF et al. (1990), Neuropsychological assessment of monozygotic twins discordant for schizophrenia. Arch Gen Psychiatry 47(11):1066-1072

Heaton, R. K., Si Crowley, T. J. (1981). Effects of psychiatric disorders and their somatic treatment on neuropsychological test results. In S. B. Filskov \& T. J. Boll (Eds.), Handbook of clinical Neuropsychology, 481 - 525.

Hogarty, G.E., \& Flesher, S. (1999). Developmental theory for a cognitive enhancement therapy for schizophrenia. Scizophrenia Bulletin 25: 677-92.

Keefe. R S; Hawkins. K. (2005). Assessing and Treating Cognitive Deficits Psychiatric Times; 22(3).

Keefe RS, Goldberg TE, Harvey PD et al. (2004), The Brief Assessment of Cognition in Schizophrenia: reliability, sensitivity, and comparison with a standard neurocognitive battery. Schizophr Res 68(2-3):283-297.

Keefe RSE, Eesley CE, Poe M (2014), Defining a cognitive decrement in schizophrenia. Biol Psychiatry.

Keefe RSE, Gold JM (2013), Comparing pharmacologic strategies for improving cognition. In: Improving Cognitive Function in Schizophrenia, 2nd ed., Keefe RSE, ed. London: Science Press, Ltd.

Kremen WS, Seidman LJ, Faraone SV et al. (2000), The paradox of normal neuropsychological 


\section{Cognitive Impairment in Schizophrenia: An overview of assessment and management}

function in schizophrenia. J Abnorm Psychol 109(4):743-752.

Kolb B, Whishaw IQ (1983). Performance of schizophrenic patients on tests sensitive to left or right frontal, temporal, or parietal function in neurological patients. J Nerv Ment Dis 171: 435-443.

Kurtz, M. M., Moberg, P. J., Gur, R. C., and Gur, R. E. (2001). Approaches to cognitive remediation of neuropsychological deficits in schizophrenia: A review and metaanalysis. Neuropsychology Review, 11: 197- 210.

Lehman, A F. (1995). Vocational rehabilitation in Schizophrenia. Schizophrenia Bulletin, 21(4): 645-656.

Muller, B.W., Sartory, G \& Bender, S. (2004). Neuropsychological deficits and concomitant clinical symptoms in schizophrenia. European Psychologist. Vol 9 (2), 96- 106.

Neuchterlein, K. H. (1977). Reaction time and attention in schizophrenia: Acritical evaluation of data and theories. Schizophrenia Bulletin 3: 373- 428.

Palmer BW, Heaton RK, Paulsen JS et al. (1997), Is it possible to be schizophrenic yet neuropsychologically normal? Neuropsychology 11(3):437-446.

Pilling, S., Bebbington, P., Kuipers, E., Garety, P, Geddes, J., Matrindale, B, Orbach, G., and Morgan, C. (2002). Psychological treatments in schizophrenia: II. Meta analyses of randomized controlled trials of social skills training and cognitive remediation. Psychological Medicine, 32: 783- 791.

Saykin AJ, Gur RC, Gur RE et al. (1991), Neuropsychological function in schizophrenia. Selective impairment in memory and learning. Arch Gen Psychiatry 48(7):618-624.

Saykin, A. J., Shtasel, D. L., Gur, R. E., Kester, D. B., Mozley, L. H, Stafiniak, P., and Gur, R. C. (1994). Neuropsychological deficits in neuroleptic naïve patients with first- episode schizophrenia. Archives of General Psychiatry 51: 124- 131.

Sharma, T., \& Antonova, L. (2003) Cognitive function in schizophrenia. Deficits, functional consequences, and future treatment. Psychiatric Clinics of North America, 26: 25- 40.

Sitskoorn MM, Aleman A, Ebisch SJ et al. (2004), Cognitive deficits in relatives of patients with schizophrenia: a meta-analysis. Schizophr Res 71(2-3):285-295.

Twamley, E. W., Jeste, D. V., and Bellack, A. S. (2003). A review of cognitive training in schizophrenia. Schizophrenia Bulletin, 29 (2): 359- 382.

Weinberger DR, Berman KF, Zec RF (1986). Physiologic dysfunction of dorsolateral prefrontal cortex in schizophrenia. I.Regional cerebral blood flow evidence. Arch Gen Psychiatry 43: $114-124$. 